\title{
ANALYSIS OF BEAM-INDUCED DAMAGE TO THE SLC POSITRON PRODUCTION TARGET*
}

\author{
Vinod K. Bharadwaj, Yuri K. Batygin, John C. Sheppard, David C. Schultz, P.O. Box 4349, SLAC, \\ Stanford, CA 94309, USA \\ Stan Bodenstein, James Gallegos, Robert Gonzales, James Ledbetter, Manuel Lopez, Robert \\ Romero, Tobias Romero, Robert Rutherford, Stuart Maloy, LANL, P.O. Box 1663, Los Alamos, \\ NM 87454, USA
}

\begin{abstract}
The nominal Next Linear Collider (NLC) positron production design is based on extrapolation of the existing SLAC Linear Collider (SLC) positron production system. Given that the SLC positron production target failed during a run, it is necessary to analyze the beam-induced damage to the target in order to validate the extrapolations on which the NLC target is based. The failed SLC target and its associated housing were sent to existing "hot-cell" facilities at LANL for analysis. The target material, a tungsten-rhenium "puck", was removed from the housing and photographed and x-rayed. Leak-checking on the cooling system was performed. Sections were then removed from the target to determine the extent of internal damage to the material. High resolution photographs were taken and extensive hardness tests were performed on the irradiated and non-irradiated areas of the target material. The results of these analyses and conclusions applicable to the NLC target design are presented in this paper.
\end{abstract}

\section{INTRODUCTION}

The SLC at the Stanford Linear Accelerator Center (SLAC) is a positron-electron linear collider running at approximately $100 \mathrm{GeV}$ in the center-of-mass. It uses a 30 $\mathrm{GeV}$ electron drive beam incident on a target made of $\mathrm{W}$ $26 \mathrm{Re}$, a tungsten-rhenium with $26 \%$ rhenium, to produce the needed positrons [2]. The target material is a disk 2.75 inches in diameter and six radiation lengths (.812 inches) thick. The disk is water-cooled using stainless steel tubes in a silver matrix. The target is trolled at $0.5 \mathrm{~Hz}$ so that the drive beam traces out a circular path around the W-Re material. In 1998, the SLC positron target failed after six years of operation. This failure manifested itself as a small leak in the target vacuum and reflected power trips in the capture RF immediately downstream of the target. Investigation of the failure mode in this target was deemed critical to improved designs of future positron targets (such as the target needed for the NLC project). The failed SLC target and its associated housing were shipped to the CMR Wing 9 hot cells at the Los Alamos National Laboratory (LANL) where facilities exist for the analysis of highly radioactive and contaminated materials.

\section{TARGET ANALYSIS}

The target analysis proposal consisted of three phases. The first phase consisted of removing the actual target material from its housing. The second phase consisted of non-destructive tests such as leak checking and taking Xrays photographs. The third phase was to slice the target and polish it for metallurgical examination and hardness testing.

\subsection{Leak Check}

After the target arm was removed from its housing, the target cooling water lines were pressurized with helium to 10 psi (see Fig. 1) and Snoop liquid leak detector fluid was applied to both sides of the target. A small leak was observed on the exit side of the target.

Figure 1: SLC Positron Target Leak Checking Setup

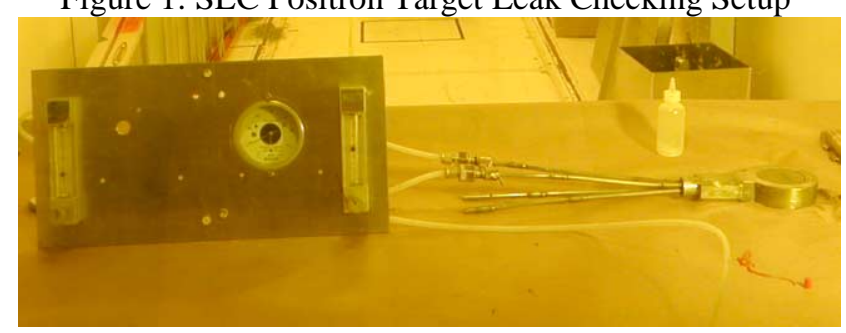

\subsection{X-ray and Flow test}

The target was X-rayed to look for blockages in the stainless steel water cooling lines. No significant blockage was observed. The X-ray photographs did not show any conclusive evidence of cracks in the cooling tubes.

\subsection{Optical Microscopy and Slicing Method}

Next, the target was transferred to a hot cell containing a microscope and photos at magnifications up to $20 \mathrm{X}$ were taken. Photos of the beam entry face of the target are shown in Figs. 2a-b and the beam exit face in Figs. 3a-b. The beam entry face showed areas in the silver with a dendritic solidification pattern. This area corresponded to the top of the silver casting after it was machined to size. The beam exit face showed a circular crack where the beam exited through the W-26Re. Additional cracking was present in the silver especially in areas where the beam was closer to the W-26Re/Silver interface.

*Work supported by Department of Energy, contract DE-AC03-76SF00515. 


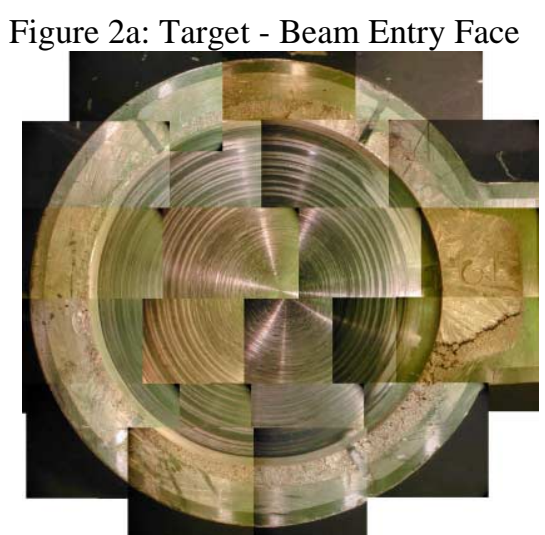

Figure 2b: Target - Beam Entry Face Detail

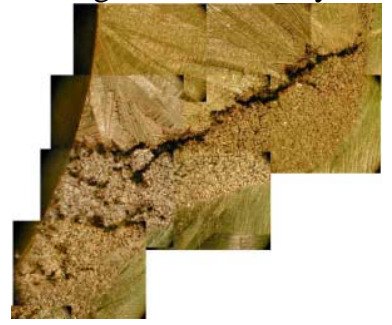

Figure 3a: Target - Beam Exit Face

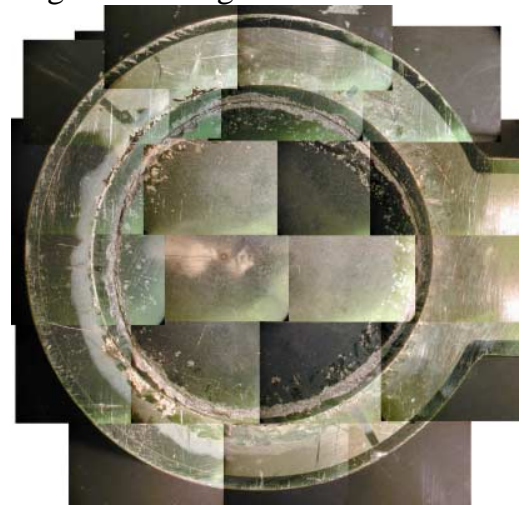

Figure 3b: Target - Beam Exit Face Detail

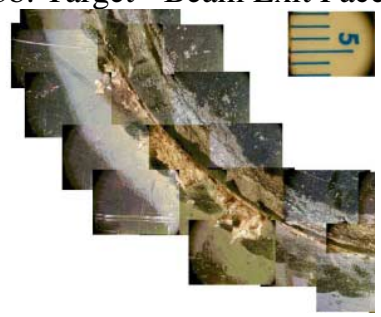

These optical micrographs were used to determine the locations where the target would be sliced. Fig. 4 shows the exit face of the target and the location where the cuts were made. Cut 0 went tangentially through the cooling tubes near where the leak was observed. Cut1 went radially through the location of the leak. Cut 3 was chosen to look at an area far away from the location of the leak. Cut2 was chosen to cut the wedge into two pieces for mounting. Each cut was performed with a high speed diamond cutting saw and took approximately 4-6 hours.

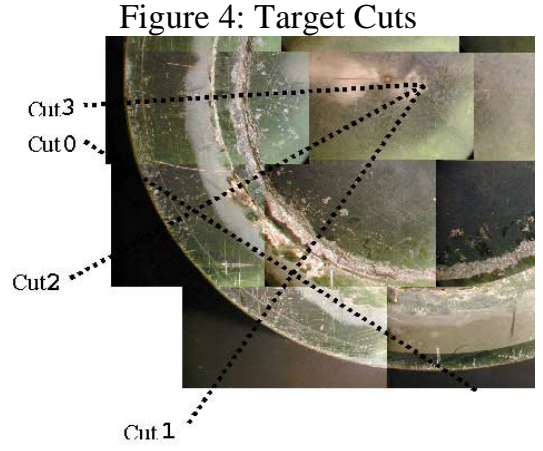

\subsection{Metallography}

The slices were cut to size and epoxied into $1.24 \mathrm{in}$. diameter mounts. The samples were ground with 600 grit sand-paper and polished down to 1 micron using diamond paste. These specimens were then observed using a Leitz metallograph at magnifications of up to 500X. No significant features were found on the pure W-26Re samples. On the other hand many interesting features were observed on Cut1 and Cut3 near the steel/silver/W26Re interfaces.

Cut1 went through stainless steel tubes, followed by silver matrix, then through a stainless steel spacer, through the silver matrix again and finally through the W26Re. Higher magnification pictures of Cut1 show two interesting areas, see Fig. 5 (right). First there are many cracks present in the tungsten-rhenium and in the silver. Second material has spalled off of the tungsten-rhenium and the silver at the edge of the specimen. It should be noted that the analysis showed no irregularities at the stainless steel water cooling tubes.

Cut3 goes through the stainless steel tubes, the silver matrix and the W-26Re. When viewing this cut using higher magnification, two features are noted in the silver. There is porosity in the silver, which seems to be associated with the dendritic cast structure. Areas in the silver at the stainless steel/silver interface show the presence of fine porosity or a second phase. Cracking was observed in the W-26Re, see Fig. 5 (left), and there is also the presence of a fine second phase on the edge of the silver.

Figure 6: Porosity and evidence of second phase in the silver
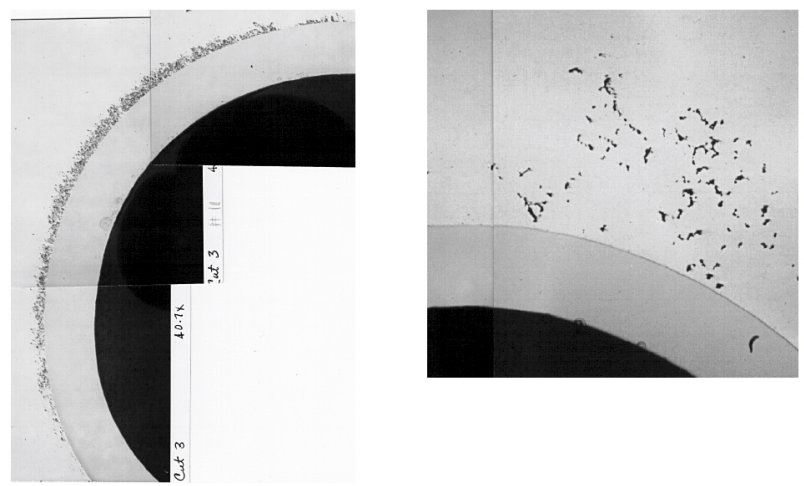


\subsection{Hardness Testing}

Diamond pyramid hardness tests were performed in the stainless steel tubes, the silver matrix and the W-26Re using a Leitz metallograph with a $400 \mathrm{~g}$ load. Autoradiographs of the polished Cut 1 and Cut 3 specimen were taken and were used for determining the locations for performing the hardness tests.

Very little change was observed in the hardness of the stainless steel and the silver matrix due to the effects of irradiation. A significant variation of hardness, however, was measured in the $\mathrm{W}-26 \mathrm{Re}$ where the electron beam irradiated the target. Hardness traces were performed making sure that each hardness indentation was a significant distance (at least five indent diameters) away from the previous indent to avoid interaction with the damage zone associated with the measurement. Figure 6 shows the variation of the hardness measured on Cut1 moving from the beam entry area toward the beam exit area near the center of the beam path. This figure shows that the hardness increases significantly (approximately twice its original unirradiated value) as one moves toward the beam exit face of the specimen (higher radiation dose). Evidence of fresh cracking was observed around an indentation made on the beam exit side of Cut1, indicating that the hardness increase is also accompanied with a decrease in ductility. The hardness also varied as one moved transversely away from the area where the beam went through the tungsten-rhenium. Figure 7 shows this variation measured on Cut 1 and Cut 3 . The hardness was measured in the location where the beam interacted with the tungsten at the height of the center of tube A along a line toward the silver/W-26Re interface.

\section{CONCLUSIONS}

The W-26Re positron target of the Stanford Linear Accelerator Center linac gave many years of reliable service. When the target is impacted by a high energy electron beam, creating the electromagnetic shower in which the positrons are produced, sudden high temperature increases create high shock and stress in the material. The target was designed to withstand these stresses. The electromagnetic shower will also cause radiation damage to the target material. This damage is evidenced by the changes in the hardness of the W-26Re in the areas where the shower occurred. The damage may have led to degradation in the material's strength, leading ultimately to the observed failure.

This finding is of particular importance to the positron source designs of future accelerators like the Next Linear Collider. These require higher positron currents that that of the SLC system. This study established the parameters for a failure mode that must be taken into account by the designers of the new systems, e.g. the NLC design is constrained to keep the peak shock at the target to the SLC levels, leading to a scheme with 3-4 targets multiplexed to produce the requisite positron flux [3].
Figure 5: Target Cracking, Cut1 (right), Cut3 (left)
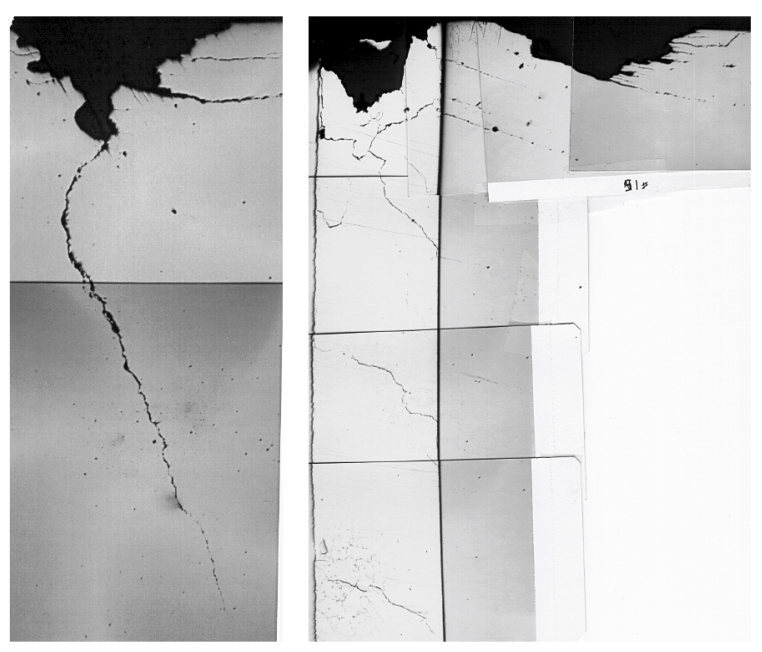

Figure 6: Hardness variation along Cut1

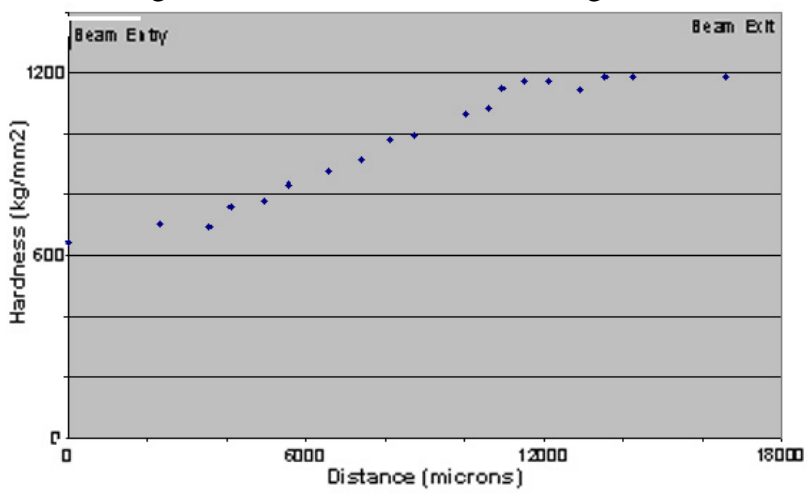

Figure 7: Hardness variation towards interface (Cut1 and Cut3)

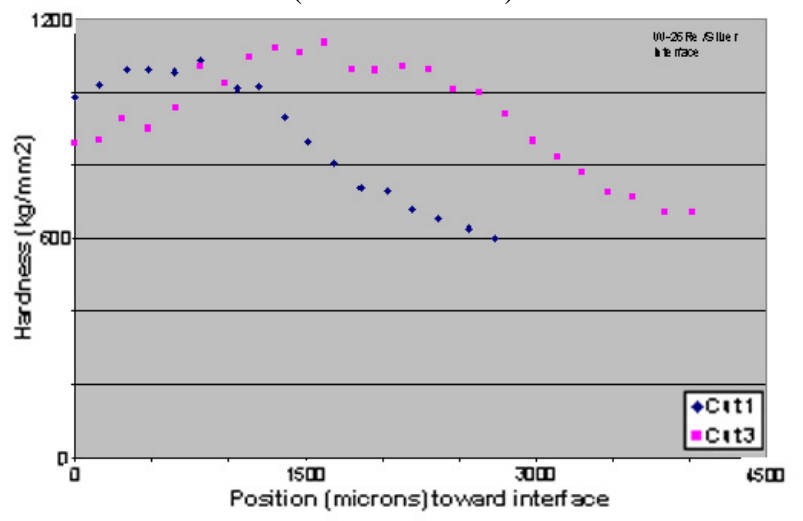

4 REFERENCES

[1] Krejcik, P., et al., 'Recent Improvements in the SLC Positron System Performance," Proc. 3rd EPAC, Berlin, 1992 (also SLAC-PUB-5786, March 1992).

[2] Maloy, S., et al., "SLC Target Analysis," LANL LA UR-01-1913, June 2001.

[3] J. C. Sheppard et al, "Update on the NLC Injector System Design", Proc. PAC 2001, Chicago, 2001 